\title{
Les systèmes industriels porcins : la mort comme travail ordinaire
}

\author{
Sébastien Mouret ${ }^{\mathrm{a}}$, Jocelyne Porcher ${ }^{\mathrm{b}}$ \\ a Doctorant en sociologie, INRA-SAD LISTO, ENESAD, BP 87999, 21079 Dijon cedex, France \\ b Zootechnicienne, INRA-SAD APT, INA-PG, 16 rue Claude Bernard, 75231 Paris cedex 05, France
}

La relation entre les hommes et les animaux est dans notre société une question qui fait de plus en plus débat, que ce soit à l'occasion de la crise de la "vache folle", mais aussi à propos du clonage, ou dans une sphère plus quotidienne, à propos de la question des droits des animaux et de leur statut ontologique. Cet article explore le nouveau rapport à la mort de l'animal au sein des systèmes industriels porcins, la transformation des carcasses en farines animales étant aujourd'hui impossible. Comment la mise à mort et la gestion des cadavres peuvent-elles entrer dans le quotidien de l'éleveur? Euphémisation de la violence, gestes techniques de mise à mort prétendent «humaniser », grâce à tout un panel d'outils d'euthanasie, jusqu'au compostage des cadavres évoquant un travail de jardinier.

La Rédaction

\author{
Mots-clés : \\ systèmes de \\ production \\ industriels ; travail ; \\ animaux d'élevage; \\ mort ; \\ encadrement \\ scientifique \\ et technique
}

\section{Keywords:}

industrial pig production; work; farm animals;

death; scientific and technical management

\begin{abstract}
Résumé - Le contenu du travail dans les systèmes industriels porcins se caractérise par un nombre important d'animaux à tuer et de cadavres à gérer. De nouvelles procédures - euthanasie, compostage, incinération - sont préconisées par l'encadrement scientifique et technique. À partir de l'analyse des concepts mobilisés dans la littérature scientifique et professionnelle française et nord-américaine, nous montrons que ces procédures s'appuient sur une euphémisation de la violence et sur une occultation de la réalité de la mort des animaux. L'euthanasie repose sur une souffrance animale objectivée qu'il s'agit, via la médiation d'outils ad hoc, de supprimer. La gestion des cadavres est fondée sur une conception végétalisée du cadavre comme matière à transformer et «hygiéniser». Ce travail de mort soulève de très graves questions quant au rapport moral des hommes aux animaux d'élevage dans ces systèmes.
\end{abstract}

\begin{abstract}
Industrial pig production: death as routine work. Killing unproductive animals and managing their corpses makes up an increasing part of work in industrial pig production. Until now these tasks were carried out by slaughterhouses. But the situation is changing. In industrial systems scientists and technicians are incorporating death as part of work. They produce concepts and tools so that workers can do their killing job "cleanly". New procedures such as euthanasia, composting and incineration have been introduced to this end. A review of scientific and technical papers and analysis of the concepts used enabled us to show that these procedures are based on the euphemization of violence and occultation of animal death. By describing animal pain as a biological problem which can be measured independently of other considerations, slaughter of unproductive animal may be constructed as "euthanasia". Worker suffering is thus mitigated since "euthanasia" is in fine considered merciful to animals. Corpse management is based on corpse construction as raw material in which animal raw material is considered similar to plant raw material: both refer to nature, i.e. to inevitable death and decomposition. The role of machinery and workers is simply to clean this animal raw material to produce hygienic, safe, useful and profitable "compost". This death work raises serious questions relative to animal welfare. Can death be an innocent part of work for farmers and workers?
\end{abstract}

Le contenu du travail des éleveurs et des salariés dans les systèmes de production animale met en évidence les transformations radicales qui ont été générées

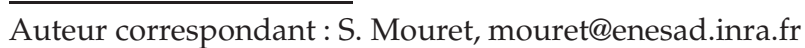

par l'industrialisation de l'élevage. La mort des animaux, notamment en systèmes industriels, y occupe une place croissante. Jamais en France, semble-t-il, le nombre d'animaux morts, le travail d'abattage des animaux mourants et la gestion de leurs cadavres n'ont été aussi présents 
dans le quotidien des éleveurs et des salariés ${ }^{1}$. Le taux de pertes moyen en maternité ${ }^{2}$ fourni par $1^{\prime}$ IFIP $^{3}$ montre qu'après une baisse faible mais constante de $1970(23,1)$ à 1994 (17,7), il s'élève depuis régulièrement (21,1 en 2005). Le taux de pertes "sevrage-vente », qui était de 5,3 en 1977 et de 5,4 en 1994, avec des variations étroites entre ces deux années, est de 7,2 en 2005. Pour un élevage porcin moyen de 160 truies de type naisseur-engraisseur, les pertes s'élèvent à plus de 1330 animaux par an (y compris les mort-nés), soit plus de 15,3 tonnes de cadavres de porcs par an, soit une production annuelle de $96 \mathrm{~kg}$ de cadavres par truie, soit, pour le troupeau français d'environ 1,3 million de truies, une production annuelle de 131200 tonnes de cadavres de porcs (Chevillon et al., 2005).

L'histoire de la filière porcine industrielle, telle qu'elle est restituée par le discours des éleveurs et des salariés, témoigne de leur lutte constante contre la maladie et la mort. Ainsi que l'exprime un éleveur installé en 1968, «en post-sevrage, j'ai perdu jusqu'à $30 \%$ de porcelets, mais économiquement, on n'a jamais souffert... J'ai eu énormément de cochons à crever, on était tous des apprentis sorciers. Il y en avait un qui voulait trouver son virus, l'autre qui voulait ceci, l'autre qui voulait cela... » $\mathrm{La} \mathrm{MAP}{ }^{4}$, ainsi que les nombreux virus récurrents dans les systèmes industriels, déciment aujourd'hui encore les animaux. Toutefois, les conséquences économiques pour les éleveurs sont bien plus dramatiques et la confrontation avec la mort, du fait de l'augmentation de la taille des exploitations, a pris une autre ampleur. Les exploitations «spécialisées porc », qui représentent $18 \%$ des exploitations porcines en 2000 contre $5 \%$ en 1988 (Ilari et al., 2004), concentrent en effet la moitié du cheptel porcin français. Pourtant, tous ces chiffres ne sauraient rendre compte de l'importance réelle du travail lié à la mort des animaux pour les travailleurs : l'euthanasie des animaux et la gestion de leurs cadavres.

L'internalisation de la mise à mort des animaux qui ne répondent plus aux standards de production et aux normes d'abattage des abattoirs et, à moyen terme, de la gestion de leurs cadavres sur l'exploitation par les travailleurs, s'inscrit dans le processus d'industrialisation de la production porcine mis en place à partir du plan de rationalisation de la production en 1970. La destinée

\footnotetext{
${ }^{1}$ Cet article s'inscrit dans le cadre d'une thèse sur la place de la mort des animaux dans le travail en élevage porcin que réalise actuellement Sébastien Mouret.

2 Pertes sur nés totaux, c'est-à-dire nombre de porcelets morts / nombre de porcelets nés. Ce chiffre, selon les travailleurs, sous-estime la mortalité réelle, car il ne comptabilise pas dans les «nés » les porcelets chétifs, en nombre croissant du fait de l'hyperprolificité des truies. Les travailleurs doivent achever ces animaux.

${ }^{3}$ Institut de la filière porcine (ex-Institut technique du porc).

${ }^{4}$ Maladie d'amaigrissement du porcelet, apparue vers 1996 dans les systèmes industriels.
}

des animaux «mal à pied », jusqu'à récemment prise en charge par les abattoirs, est aujourd'hui abandonnée aux éleveurs. C'est également sur leurs épaules que tend actuellement à reposer une partie de l'équarrissage, dont la répartition du coût au sein de la filière est aujourd'hui reconsidérée, les pouvoirs publics se désengageant progressivement.

C'est parce que la mort des animaux devient effectivement un travail dans ces systèmes que des techniques sont élaborées et prescrites aux travailleurs par leur encadrement scientifique et technique (Porcher, 2003a). Ce travail de mort interroge l'évolution du sens du métier d'éleveur du point de vue du rapport à la vie animale et la manière dont des attentes sociales en termes de «bienêtre animal » sont anticipées et traduites en normes techniques et éthiques en fonction d'une souffrance animale objectivée. La production de ces nouvelles techniques et leur acceptabilité s'appuient sur une euphémisation de la violence de la mise à mort des animaux et de la réalité de l'animal mort, afin qu'elles soient le plus indolores et le plus "propres » possible, pour les animaux comme pour les travailleurs. Ainsi, le terme « euthanasie » cache qu'elle n'en est pas une, notamment parce que la rationalité économique est à la racine de la décision bien davantage que la compassion ou des raisons d'ordre moral. La vie animale n'a aucune valeur morale du point de vue de l'organisation scientifique du travail. De la même façon, le « compostage » des cadavres permet d'occulter le charnier ${ }^{5}$.

Nous examinerons les notions et les concepts - euthanasie, souffrance animale, éthique, compostage, nature, hygiène - mobilisés pour formuler les prescriptions relatives à ce travail mortifère et les recommandations faites aux éleveurs et aux salariés en production porcine industrielle dans l'usage de nouvelles techniques pour tuer des animaux «mal à pied »- matador, pince électrique, dioxyde de carbone - et gérer des cadavres de porcs - plateforme de compostage, incinérateur - sur l'exploitation.

À partir de l'analyse de revues et d'articles scientifiques et techniques français et nord-américains en production porcine depuis 1995, nous présenterons les nouveaux outils de ce travail de mort; puis, après avoir étudié le contexte d'évolution réglementaire, nous montrerons comment la technique tout autant que les mots participent du processus d'euphémisation.

\section{Les nouveaux outils du travail de mort en systèmes industriels porcins}

Afin de faciliter la réalisation de tâches pour lesquelles ils ne sont pas préparés et sont encore insuffisamment équipés, de nouveaux outils d'euthanasie et de gestion des cadavres de porcs sont proposés aux travailleurs

\footnotetext{
5 «Lieu où sont entassés les cadavres » (Le Petit Robert).
} 
en systèmes porcins par leur encadrement technique. Ces techniques, actuellement en développement en France via l'IFIP, sont déjà utilisés aux États-Unis et, de façon plus récente, au Québec.

L'euthanasie des animaux improductifs prend modèle sur les techniques de mise à mort en abattoir. Elles remplacent ou complètent des pratiques déjà mises en œuvre par les éleveurs et les salariés en production porcine : «assommage» par choc contre une paroi, «assommage » à la masse, etc. L'euthanasie des animaux peut désormais être pratiquée sur l'exploitation avec une pince électrique composée de deux électrodes que «l'opérateur » doit placer au niveau des yeux de l'animal, puis au niveau du cœur. Elle peut également être réalisée à l'aide d'un matador, pistolet à tige qui perfore la boîte crânienne de l'animal, ou d'un caisson étanche saturé à $70 \%$ minimum de dioxyde de carbone $\left(\mathrm{CO}_{2}\right)$. « L'équipement nécessaire est : une bouteille de gaz au $\mathrm{CO}_{2}$ sous pression, un détendeur, un réchauffeur de gaz, un analyseur de gaz, un caisson étanche » (Chevillon et al., 2004, p. 22).

Une fois ces animaux improductifs morts, la gestion de leurs cadavres peut se faire in situ par décomposition sur une plateforme de compostage ou par incinération. Cela permet de gérer l'évolution du nombre de cadavres de porcs dans les exploitations et d'appuyer la logique de concentration et d'agrandissement des unités de production.

Le compostage permet de dégrader la matière organique en présence d'oxygène. Le processus de compostage de cadavres comporte trois grandes étapes (Pigeon et Fortier, 2004a). Tout d'abord, le remplissage des cellules de l'unité de compostage : il s'agit de constituer une couche de base à partir d'un substrat, sur laquelle sera déposée une couche de cadavres. Vient ensuite «l'échauffement primaire ", ou fermentation, auquel succède «l'échauffement secondaire", encore appelé phase de maturation. L'humidité, la température, la présence d'oxygène à l'intérieur du «tas » et le « rapport du carbone à l'azote de la masse à composter » doivent être contrôlés pour que les microorganismes fassent leur travail dans des conditions idéales. La durée de la décomposition dépend de la taille des cadavres et du substrat utilisé.

Les incinérateurs comportent deux chambres de combustion (Blanchard, 2002 ; Pigeon et Fortier, 2004b). Dans la chambre primaire, les cadavres sont placés à une température de combustion de $850{ }^{\circ} \mathrm{C}$. Les gaz et les particules générés par l'incinération sont ensuite dirigés vers la chambre de combustion des gaz à $1000{ }^{\circ} \mathrm{C}$, ou chambre secondaire, afin d'y être oxydés de façon plus poussée. La capacité de chargement d'un incinérateur varie de $220 \mathrm{~kg}$ à $450 \mathrm{~kg}$ de cadavres. Un cycle d'incinération a une durée moyenne de 5 à 7 heures.
L'euthanasie et la gestion des cadavres de porcs sont, en tant que telles, un travail nouveau pour les éleveurs et les salariés. L'animal vivant et l'animal mort entrent dans un processus de production impliquant des machines, des opérateurs, des tâches, des procédés techniques qui, pour la gestion des cadavres, résultent de plusieurs années de recherches scientifiques (Larson, 2006). La valorisation potentielle des sous-produits de la transformation - farines et compost peuvent servir à la fabrication de ciment et à l'amendement des terres agricoles -, qui implique une évolution du cadre réglementaire, permet de rentabiliser ce qui était jusqu'alors un facteur limitant de la productivité. Le cadavre n'est plus une perte « sèche » pour les producteurs.

\section{Le contexte d'évolution réglementaire}

Afin de comprendre l'émergence de ces nouvelles procédures du travail prescrit, il convient de préciser le contexte d'évolution du cadre réglementaire dans lequel elle s'inscrit. Nous reviendrons d'abord sur le cas de l'euthanasie, pour ensuite aborder celui de l'internalisation de la gestion des cadavres de porcs au sein des unités de production porcine.

Le processus de rationalisation du travail a non seulement des effets sur l'organisation du travail dans les abattoirs, mais également, en amont, sur celle des unités de production de porcs. Afin d'améliorer la fluidité de leur processus de production et de ne pas pénaliser leur productivité, les abattoirs sont autorisés à refuser les animaux « en état de mort apparente, morts de maladie ou $\mathrm{d}^{\prime}$ accident ou en état de misère physiologique ${ }^{6} »$. Parmi ces animaux, certains n'ont en fait qu'un problème de mobilité dû à leur inactivité forcée. Les travailleurs, déjà contraints de tuer eux-mêmes ou de laisser à l'abandon des animaux condamnés à mourir suite à des pathologies ou à des blessures, doivent désormais également prendre en charge l'euthanasie des animaux refusés - ou susceptibles de l'être - par les abattoirs pour des raisons techniques et économiques.

Jusqu'à ces dernières années, seul le vétérinaire était habilité à pratiquer l'euthanasie des animaux d'élevage. Ce cadre réglementaire et les coûts financiers qui y étaient liés conduisaient les éleveurs, dans les faits, à se passer du vétérinaire (Joly, 1999). L'animal était abattu avec les moyens du bord, par pendaison par exemple, ou abandonné à son sort. La mise en œuvre de techniques d'euthanasie passe aujourd'hui par une évolution du cadre juridique actuel, afin que les travailleurs soient reconnus responsables, c'est-à-dire qu'ils puissent décider de l'euthanasie des animaux malades et blessés et la pratiquer eux-mêmes. Or, ces nouveaux outils n'ont pas encore

\footnotetext{
${ }^{6}$ Arrêté du 9 juin 2000 du ministère de l'Agriculture et de la Pêche.
} 
été validés officiellement par les organismes compétents. Armer les travailleurs contre leurs animaux, c'est en effet les armer potentiellement contre tout un chacun, à commencer par eux-mêmes en cas d'accident; et cela, on s'en doute, n'est pas sans poser problème. C'est pour ces raisons précisément que l'euthanasie des animaux était, antérieurement, obligatoirement pratiquée par un vétérinaire. L'évolution du cadre juridique vise à définir et à faire adopter de "bonnes pratiques d'euthanasie » en élevage, via des formations qui, prenant en compte à la fois l'impréparation des travailleurs et la question du bien-être animal, donneraient également une dimension morale à ce travail.

Du point de vue de la réglementation française et européenne, les cadavres de porcs sont définis comme des "déchets de sous-produits animaux ». L'arrêté du 7 août 1998 relatif à l'élimination des cadavres d'animaux ${ }^{7}$ interdit de jeter ou d'incinérer en quelque lieu que ce soit les cadavres d'animaux ou les lots d'animaux morts dont le poids est supérieur à $40 \mathrm{~kg}$. Les détenteurs de "sousproduits animaux », en l'occurrence les éleveurs, doivent les mettre à disposition du service public d'équarrissage (SPE) à titre gratuit. La collecte et l'élimination de ces déchets impropres à la consommation humaine sont une mission de service public assurée par les équarrisseurs qui les transforment en graisses et farines animales. Or, depuis quelques années, le SPE a perdu son équilibre financier. Suite à la crise de 1'ESB en 1996, les graisses et les farines animales ont été interdites en France dans 1'alimentation animale, mais également dans les supports et les matières fertilisantes destinées aux grandes cultures, ce qui n'est pas le cas dans d'autres pays de l'Union européenne (Lapasin, 2005). Les débouchés économiques pour les protéines animales transformées sont donc limités. De plus, le coût lié à la collecte et à l'élimination des animaux morts pour les entreprises d'équarrissage a augmenté depuis plusieurs années en raison de l'accroissement du nombre d'animaux morts dans les systèmes industriels, constant depuis 1995.

Le déséquilibre financier du SPE représente une charge financière importante pour la filière porcine française et l'État. Une réforme du SPE est donc engagée, concernant ses modalités de financement et de fonctionnement. L'État semble vouloir limiter, voire supprimer, sa participation financière au fonctionnement de ce service public. Au Québec, le retrait de l'État du service d'équarrissage a déjà entraîné une forte concentration des entreprises dans ce secteur. Les éleveurs sont confrontés à une augmentation des coûts d'équarrissage et à un éventuel refus de la part des entreprises de collecter les cadavres, notamment pour des raisons d'éloignement géographique trop important. Le problème de l'équarrissage

\footnotetext{
7 Journal officiel du 20 août 1998. Article L226-2 et suivant du
} Code rural. s'est ainsi étendu à l'ensemble des productions animales. Certains éleveurs abandonnent des cadavres d'animaux en dehors de leurs exploitations, dans des sous-bois et des cours d'eau notamment.

Afin de réduire les coûts liés au traitement des cadavres de porcs et de permettre aux producteurs porcins de se «défaire » de leurs animaux morts, la filière porcine recherche actuellement des alternatives techniques au SPE. Une meilleure rationalisation de la collecte associée à un stockage au froid des « sous-produits animaux » est pour l'instant la solution envisagée pour faire face à ce «sujet chaud» (Hamelin, 2005a). Dans une perspective d'évolution de la loi française, l'incinération, le compostage des cadavres d'animaux et la décomposition bactérienne et enzymatique sur le site de l'exploitation sont également envisagés.

\section{Euphémisation de la violence de la mise à mort des animaux}

L'emploi de la notion d'euthanasie en systèmes industriels pour nommer les procédures de mise à mort des animaux d'élevage improductifs appelle une attention particulière. Les procédés de banalisation et d'euphémisation de la violence de la mise à mort des animaux par l'euthanasie s'appuient sur une justification morale de l'acte de tuer et une réduction de la souffrance visible de l'animal mis à mort.

\section{Justifier l'euthanasie d'un point de vue moral}

Du point de vue de l'organisation du travail prescrit en production porcine, l'euthanasie est définie comme une "procédure d'élevage » qui relève d'une « démarche décisionnelle » de la part de "l'opérateur », en l'occurrence l'éleveur ou le salarié (Joly, 1999). Au cours de cette démarche, le travailleur doit «évaluer les chances de survie de l'animal», «apprécier les souffrances endurées par l'animal » et, enfin, réaliser « un raisonnement économique » qui lui permettra d'estimer les «chances de l'animal de devenir une valeur économique acceptable pour le client ». Dans le cas contraire, l'animal devient une «non-valeur » et peut donc être euthanasié.

$\mathrm{Au}$ Canada, où ces techniques d'euthanasie sont déjà appliquées, la notion d'éthique est mobilisée pour justifier leur mise en œuvre et inciter les éleveurs à euthanasier les animaux au nom de leur bien-être : "L'euthanasie doit être effectuée d'une manière qui réduise la peur et l'anxiété chez l'animal. Selon les bonnes pratiques d'élevage, les producteurs ont la responsabilité éthique et morale d'assurer une mort respectueuse aux animaux dont ils ont le soin, sans leur causer de souffrances additionnelles » (Rietveld, 2003a et b). En France l'injonction éthique faite aux travailleurs n'est pas formulée aussi 
explicitement pour justifier l'acte d'euthanasie en production porcine (Joly, 1999). L'euthanasie s'inscrit plutôt dans le cadre du bien-être des animaux d'élevage : elle a pour but de soulager l'animal de ses souffrances en lui donnant la mort.

L'euthanasie prescrite des animaux d'élevage est justifiée d'un point de vue moral par un rapport entre souffrances évitées aux animaux et avantages économiques pour les éleveurs et les consommateurs. On peut qualifier cette position de conséquentialiste ${ }^{8}$. Néanmoins, du point de vue de la zootechnie porcine, la vie animale est loin de posséder une valeur morale. Elle conserve une valeur instrumentale. La hiérarchisation des critères économiques et d'évitement des souffrances à l'animal n'est pas précisée dans la « démarche décisionnelle » d'euthanasie telle qu'elle est prescrite. Cet acte peut devenir un moyen de sélectionner les "sujets indésirables » d'un point de vue économique pour les unités de production comme pour les abattoirs. Les souffrances inutiles, que l'euthanasie prescrite prétend éviter, reposent sur une définition minimaliste de la vie animale qui ne prend pas en compte sa dimension subjective (Dantzer, 2001). Le porc industriel reste donc un «animal-machine » (Larrère et Larrère, 2001) qu'il faut arrêter pour cause de dysfonctionnement. La souffrance n'est qu'un problème mécanique dont la résolution n'impose aucune limite morale quant aux moyens à mettre en œuvre : pince électrique, $\mathrm{CO}_{2}$, etc.

Ce cadre éthique minimaliste vise surtout à justifier l'euthanasie dans un contexte où la profession porcine connaît, d'une part, une crise de sa légitimité sous l'effet des critiques morales relatives au traitement industriel des animaux d'élevage et, d'autre part, une forte désertion du salariat sous le poids croissant du «sale boulot ». $\mathrm{Du}$ point de vue des systèmes de production animale, l'animal reste une simple matière à produire. La vie animale en tant que vie vécue est déniée (Porcher, 2003b). Dès lors, comment les travailleurs peuvent-ils relier cette injonction à l'éthique, qui implique que l'animal existe officiellement - puisqu'il faut le tuer, c'est qu'il est en vie -, et l'organisation prescrite du travail qui prétend qu'il n'existe pas? Ainsi que l'écrit Jankélévitch (1997), il faut être «non- vivant», et donc déjà mort, pour ne pas mourir.

\section{Produire une mort douce}

Dans les abattoirs industriels, l'organisation du travail a, théoriquement, intégré des préoccupations

\footnotetext{
${ }^{8}$ Le conséquentialisme est une théorie en philosophie morale qui pose qu'un acte est susceptible d'être approuvé pour autant que ses conséquences contribuent à la réalisation d'un bien au sens moral du terme. L'admissibilité morale d'un acte, d'une conduite, dépend de sa contribution à la production d'un bénéfice, d'un avantage, d'un plaisir ou à l'évitement d'un dommage, d'une souffrance (Goffi, 1994).
}

sociales et réglementaires relatives au bien-être des animaux d'élevage en conciliant deux notions antinomiques : 1 'industrialisation, qui repose sur une objectivation du vivant, et «l'humanisation", fondée sur une subjectivation du rapport aux animaux (Rémy, 2003). Les procédés d'abattage visent à éviter toute souffrance inutile aux animaux. Pour cela, une phase d'étourdissement où l'animal est rendu inconscient précède la mise à mort même. L'étourdissement est pratiqué soit au $\mathrm{CO}_{2}$, soit au matador, soit par choc électrique. La saignée se fait à l'aide d'un couteau. En système industriel porcin, l'euthanasie des animaux prend en partie modèle sur ces procédés et prétend également à une mort «humanisée». Mais, par un glissement de leur fonction, le matador, la pince électrique et le $\mathrm{CO}_{2}$ ne sont plus utilisés pour « étourdir» l'animal, mais pour le tuer.

L'euphémisme «euthanasie » construit son efficacité supposée à partir de deux niveaux d'évitement : la souffrance visible de l'animal; le sang. Le premier niveau porte sur la réduction de la souffrance de l'animal mis à mort au regard de critères objectivables définis par les concepteurs des techniques d'euthanasie. Les choix et les mises au point des techniques d'euthanasie visent à minimiser l'expression de la souffrance médiatisée par ces critères : cris, spasmes, débattements. Il s'agit d'obtenir une mort « rapide et efficace» (Chevillon et al., 2004), pour chaque type d'animal, grâce à une adéquation entre l'outil et le type d'animal à tuer. Pour la mise à mort des animaux de gros gabarit (porcs charcutiers, truies, verrats), l'euthanasie électrique est recommandée. Pour les porcs de petit gabarit (porcelets), c'est l'emploi du matador ou du $\mathrm{CO}_{2}$ qui est préconisé. Le second niveau porte sur l'évitement d'effusion de sang au cours de l'acte d'euthanasie : d'une part, parce que l'effusion de sang rend évidente la tuerie et la violence; d'autre part, parce que la saignée est, par définition, l'acte de "désanimation » de l'animal pour la préparation de la viande destinée à la boucherie. «La saignée fait de l'animal tout autre chose qu'un cadavre » (Vialles, 1987). Or, dans le cadre de l'euthanasie, le cadavre doit rester cadavre. Il s'agit d'obtenir une mort «propre » (Chevillon et al., 2004), qui réponde à la logique d'humanisation dans laquelle l'euthanasie des animaux d'élevage prétend s'inscrire.

Toutefois, la mise à mort est loin d'être douce et sans souffrance pour les animaux, même si, pour les travailleurs eux-mêmes, il est important qu'elle soit décrite et perçue comme telle pour qu'elle ait un sens et qu'ils puissent la pratiquer. Au regard des expérimentations menées sur l'euthanasie des porcs (Chevillon et al., 2004), les méthodes et les outils employés ne semblent pas être en mesure de supprimer totalement la souffrance de l'animal lors de l'euthanasie. La présence de cris, de spasmes et de débattements pendant quelques instants montre l'existence d'une souffrance « résiduelle». L'animal résiste à la maîtrise de la 
connaissance scientifique et technique, ce qui, du même coup, empêche que l'euphémisme " euthanasie » s'installe dans l'esprit des travailleurs en production porcine industrielle. La souffrance objectivée par les concepteurs des procédures correspond à la douleur ressentie par l'animal malade, blessé puis mis à mort, mais non à la souffrance qu'il peut éprouver avant et au cours de cet acte. Car la prise en compte de la subjectivité de l'animal (Straus, 2000) est absente de la conception scientifique de la vie animale dont dépend l'euthanasie prescrite. Ces minutes peuvent être perçues comme très longues et sont cause de souffrance pour des « opérateurs » déjà contraints de se défendre psychologiquement contre la souffrance liée aux conditions de vie au travail dans les systèmes industriels (Mouret, 2005 ; Porcher, 2003a). Le travail dans les systèmes industriels semble présenter les caractéristiques du "sale boulot », lequel joue un rôle négatif du point de vue de l'accomplissement de soi, de l'identité.

Le «sale boulot » renvoie d'abord aux tâches considérées comme "physiquement dégoûtantes ou symbolisant quelque chose de dégradant et d'humiliant » (Hughes, 1996). Elles confrontent l'homme dans son travail à des dimensions taboues de l'expérience humaine, comme l'impur, l'infect, la maladie et la mort. Comme le souligne Salmona (1985), la maladie et la mort des animaux en élevage représentent des « ombres des travailleurs du corps ». La dimension mortifère du travail suscite chez les éleveurs des peurs liées à des risques réels ou fantasmatiques. Le «sale boulot» correspond également «à ce qui va à l'encontre de nos conceptions morales les plus héroïques » (Hughes, 1996). Si la construction scientifique de l'organisation du travail prescrit en production porcine prétend à un traitement respectueux et «naturel » des animaux d'élevage vivants et morts, on peut s'interroger sur l'existence et l'expression d'un rapport moral à la vie et à la mort des animaux chez les travailleurs euxmêmes, notamment sur la façon dont ce «sale boulot » est perçu et sur la manière dont ce rapport moral peut être construit ou déconstruit.

\section{Euphémisation de la réalité des cadavres d'animaux}

En Amérique du Nord comme en France, les acteurs de la profession porcine sont confrontés à des critiques relatives à la logique industrielle de production, à l'impact des pratiques d'élevage sur les ressources environnementales et au traitement des animaux d'élevage. Dans un tel contexte social, l'usage dans les élevages de nouvelles techniques de gestion des cadavres s'accompagne d'explications sur leur innocuité pour la «nature » et la santé humaine, qui ont pour effet de légitimer leur utilisation. La «nature », l' « hygiène » et la «biosécurité » sont des notions fortement mobilisées par les concepteurs pour argumenter la mise en œuvre des solutions techniques alternatives au service public d'équarrissage auprès des consommateurs, des concitoyens, mais également des éleveurs et des salariés. Ces arguments font du traitement des cadavres en systèmes industriels un processus naturel et contribuent ainsi à le banaliser.

Le compost issu de cadavres d'animaux est tout d'abord comparé au «terreau » et à «l'humus » : «Le compost est un matériau stable, de couleur foncée, d'aspect rappelant le terreau ou l'humus, qui peut être épandu sur les champs » (Pigeon et Fortier, 2004a). Cette comparaison avec le végétal vise à occulter le caractère cadavéreux du compost produit à partir d'animaux morts. L'animal « végétalisé » perd ainsi sa charge affective. Il ne renvoie plus à la mort humaine, il ne fait plus peur.

Le compostage des cadavres de porcs est ensuite présenté comme une technique de production "naturelle». «Le compostage est un processus naturel au cours duquel des matières organiques sont décomposées par des micro-organismes, comme les bactéries et les champignons, et transformées en un produit appelé compost » (Kobel et al., 2003). L'argument s'appuie sur la nature périssable du cadavre de l'animal appelé à une décomposition décrite comme un processus naturel, comme si elle était dépourvue de toute intervention humaine; la dégradation de cadavres d'animaux serait un phénomène normal dans le monde animal : "Chargez les carcasses et les copeaux dans le cylindre, laissez chauffer, mettez sous tension quelques heures par jour et laissez faire la nature » (Hamelin, 2005b, p. 47).

D'un point de vue social, cette vision purifiée du monde naturel traduit un désir de prise en compte de la nature des choses, du respect d'un ordre naturel dans certaines de nos pratiques agricoles, fortement critiquées pour leur artificialisation. Le sentiment, partagé par les professionnels et nos concitoyens, que «nous sommes allés trop loin » témoigne de ce que certaines limites dans notre rapport à la nature ont été franchies qui n'auraient pas dû l'être. Les techniques de compostage d'animaux morts constitueraient une sorte de prise en compte de cet ordre naturel. Elles ne seraient pas liées aux systèmes de production industriels, elles ne renverraient pas à un lien de travail dégradé aux animaux d'élevage, mais au contraire à la prégnance, voire à l'immanence, de la «nature ». Dans ces représentations, le monde industriel s'articule spontanément avec la «nature » dans une sorte de vision œcuménique de notre relation de travail aux animaux.

La justification des différentes techniques de gestion des cadavres d'animaux repose également sur les notions $\mathrm{d}^{\prime}$ « hygiène », de " biosécurité », de " sécurité sanitaire » et de «normes environnementales ». L'argument principal des concepteurs est que «la biosécurité des élevages est associée au respect de l'environnement » (Hamelin, $2005 b$, p. 46). Si ces machines et ces procédés techniques 
permettent de transformer de la «matière animale » en un sous-produit valorisable d'un point de vue économique, ils permettent également d'obtenir un matériau «hygiénisé »: «L'efficacité du process est validée et sa fiabilité avérée. De plus les paramètres microbiologiques et parasitologiques du compost final permettent de le qualifier de produit «hygiénisé » [...]. [...] en période de températures moyennes positives, le Biovator met environ deux semaines pour «digérer » une truie et deux-trois jours pour un porcelet» (Viel, 2006, p. 70) ${ }^{9}$. L'ensemble de ces procédés techniques s'appuie sur un traitement thermique des cadavres de porcs. La chaleur transforme les animaux morts en cendres ou en compost. Elle permet une purification des cadavres par élimination des impuretés matérielles, c'est-à-dire des virus et des germes susceptibles de nous contaminer. «Cette dégradation [le compostage] s'accompagne d'une réduction des microorganismes pathogènes et de la désodorisation du produit » (Pigeon et Fortier, 2004a). La purification élimine la maladie et chasse ainsi la mort de notre univers familier. Elle participe au déni de la mort humaine dans nos sociétés contemporaines (Thomas, 1975). La purification par le feu est perceptible dans la manière de nommer les incinérateurs de cadavres de porcs : le Brûle-o-max, le Puratone ou encore le Max-Flam. De la même manière, la technicisation de ce travail et le langage utilisé contribuent à mettre à distance la réalité de la mort des animaux. «L'échauffement primaire » et «l'échauffement secondaire » remplacent par euphémisation le mot "putréfaction » pour nommer la décomposition d'un cadavre. Comme le souligne Jankélévitch (1997, p. 62), « la voie oblique de l'euphémisme, les cercles de la périphrase, les zigzags de la conversation sont autant de subterfuges pour esquiver le mouvement rectiligne qui désignerait, d'une désignation transitive, le complément direct appelé mort [...]. Ces babillages sont des subterfuges de $1^{\prime}$ «allégorie » et de l'euphémie : grâce à eux, l'homme effarouché par l'innommable restera en marge de la question. »

Au-delà de la «biosécurité », la purification matérielle des cadavres de porcs occulte la réalité de la mort des animaux dans le travail en systèmes industriels. Le compostage, l'incinération, entraînent une transformation physique des cadavres, qui deviennent cendres ou compost. Ces procédés techniques permettent d'éliminer, ou du moins de diminuer, la présence et l'odeur des animaux morts. Tout ce qui concrétise l'animal mort, et donc évoque sa réalité, disparaît : la vue du cadavre et son odeur. Le cadavre de porc ne sent plus, ne se voit plus. La réalité de la mort des animaux est occultée. "Compte tenu des températures obtenues, il est

\footnotetext{
${ }^{9}$ Le Biovator $^{\mathrm{TM}}$, dans sa version $12,5 \mathrm{~m}$ (de long pour 1,2 m de diamètre), coûte 45000 dollars. Dans les conditions canadiennes, précise le journal Porc Magazine, il faut moins de deux ans pour assurer le retour sur investissement.
}

considéré comme hygiénisé. Par rapport à un compost traditionnel, le système revendique une parfaite discrétion (vue et odeurs des cadavres), ainsi qu'une totale sécurité sanitaire » (Hamelin, 2005b, p. 47).

Afin de s'assurer de la discrétion la plus totale lors de la mise en place et du fonctionnement de ces équipements et procédés, certaines précautions sont recommandées aux éleveurs et aux salariés (Blanchard, 2002). Pour éviter que les bacs réfrigérés d'équarrissage et les sites de compostage d'animaux morts ne servent de garde-manger aux animaux "sauvages ", les installations doivent être équipées de grillage. Que diraient des voisins, résidant à proximité d'unités de production porcine, s'ils venaient à retrouver une patte, un groin ou un os de cochon laissés par leur animal domestique dans leur propriété ? Concernant l'incinération à la ferme, l'emplacement du bâtiment abritant l'incinérateur doit réduire les risques de perception des fumées, porteuses de particules et de différents composés, par les voisins. Il est donc recommandé aux producteurs « d'éviter de l'installer dans l'axe des vents dominants par rapport à des habitations voisines ou des lieux publics ». De plus, l'incinération doit être réalisée de "manière régulière ", surtout l'été, où le taux de mortalité dans les bâtiments augmente fortement en raison de la chaleur. Il s'agit d'éviter une accumulation de cadavres qui occasionnerait des "problèmes d'odeurs ou de contamination $»$.

\section{Conclusion}

La transformation de la relation des hommes aux animaux dans les systèmes industriels s'accompagne d'une euphémisation de la violence et de la réalité de la mort des animaux, liée à une rationalisation instrumentale du travail dans laquelle ces systèmes sont enracinés. Les procédures d'euthanasie des animaux tentent de garantir une mort «douce », sans souffrance visible, et de répondre à des justifications morales. Le compostage et l'incinération de cadavres de porcs participent de recommandations techniques qui occultent la réalité de l'animal mort dans le travail des éleveurs et des salariés en production porcine.

Du point de vue des systèmes industriels, le cochon, mort ou vivant, est réduit à l'état de matière animale que des procédés techniques fluidifient, détruisent ${ }^{10}$, transforment, recyclent. Or, si travailler c'est produire, c'est également être en relation avec autrui - en l'occurrence avec des êtres humains et des animaux d'élevage - et

\footnotetext{
${ }^{10}$ Le terme de «destruction » est utilisé de façon récurrente pour nommer l'abattage des animaux "à risques", susceptibles d'être contaminés par l'ESB ou le virus H5N1. Son usage contraste fortement avec le concept «d'euthanasie », également employé pour nommer ces pratiques d'abattage qui répondent à des mesures de santé publique.
} 
s'épanouir, construire son identité (Dejours, 2000). Au regard de l'évolution mortifère de la relation des hommes aux animaux en systèmes industriels, il paraît donc essentiel de redonner leur place aux autres rationalités du travail. De nombreux éleveurs et salariés en production porcine industrielle affirment en effet $\mathrm{qu}^{\prime}$ « on ne peut pas avoir de considérations pour les animaux ${ }^{11} »$. Comment se construit un rapport moral aux animaux d'élevage porcin du point de vue de ces acteurs? Quel est l'impact de l'organisation du travail prescrit en systèmes industriels sur ce rapport moral ? Comment la mort peut-elle devenir ordinaire pour ces travailleurs qui doivent tuer et gérer un nombre croissant d'animaux et de cadavres (Mouret, 2005)?

Dans le contexte d'un intérêt scientifique et social grandissant pour le bien-être des animaux d'élevage, l'euthanasie et la gestion des cadavres en systèmes industriels en tant que travail « ordinaire » interrogent le sens de ces tâches mortifères et leurs effets sur les travailleurs. Les représentations du métier d'éleveur et de salarié reposent encore en grande partie sur des représentations historiques de l'élevage : faire naître, élever, protéger et soigner les animaux (Porcher, 2002). Face à ce qui semble relever de la souffrance éthique (Dejours, 1998), c'est bien la question du sens moral dans le travail avec les animaux en élevage qu'il s'agit alors d'interroger.

\section{Références}

Blanchard, D., 2002. L'incinération des carcasses à la ferme, Porc Québec, juin.

Chevillon, C., Mircovich, C., Dubroca, S., Fleho, J.-Y., 2004. Euthanasie en élevage de porc, Techni-porc, 27, 4, 21-27.

Chevillon, C., Aubry, A., Rieu, M., 2005. Gestion des cadavres de porcs en France : volumes, organisation et collecte, stockage et traitement, Techni-porc, 28, 3, 3-10.

Dantzer, R., 2001. Comment les recherches sur la biologie du bien-être animal se sont-elles construites?, in Burgat, F. (Ed.), Les Animaux d'élevage ont-ils droit au bien-être?, Paris, INRA Éditions, 85-104.

Dejours, C., 1998. Souffrance en France : la banalisation de l'injustice sociale, Paris, Le Seuil.

Dejours, C., 2000 (nouv. éd. augm.). Travail, usure mentale : essai de psychopathologie du travail, Paris, Bayard.

Goffi, J.-Y., 1994. Le Philosophe et ses animaux: du statut éthique de l'animal, Nîmes, Jacqueline Chambon.

Hamelin, É., 2005a. Facture d'équarrissage. Le froid, une réponse à un sujet chaud, Porc Magazine, 388, 68-71.

Hamelin, É., 2005b. Nouveau monde, nouvelles tendances, Porc Magazine, 390, 44-47.

Hugues, E.C., 1996. Le regard et le soi [1951], in Hugues, E.C., Le Regard sociologique : essais choisis, Paris, EHESS, 75-85.

Ilari, E., et al., 2004. Les systèmes de production du porc en France : typologie des exploitations ayant des porcs, $36^{e s}$ Journées de la recherche porcine, Paris, ITP/INRA, 1-8.

\footnotetext{
${ }^{11}$ Ce constat s'appuie sur des enquêtes réalisées par nousmêmes auprès de salariés en production porcine en France et au Québec.
}

Jankélévitch, V., 1997. La Mort, Paris, Flammarion.

Joly, J., 1999. L'euthanasie dans les élevages porcins, Bulletin des GTV, 2, 137-143.

Kobel, G., Rafail, A., Morris, J., 2003. Compostage à la ferme des cadavres de bétail et de volaille. Fiche technique, Ontario, ministère de l'Agriculture, de l'Alimentation et des Affaires rurales (http://www.omafra.gov.on.ca/french/ livestock/deadstock/facts/03-084.htm).

Lapasin, C., 2005. Un potentiel de valorisation des sousproduits animaux inexploité, Le Magazine des abattoirs, 175, $12-14$.

Larrère, C., Larrère, R., 2001. L'animal, machine à produire : la rupture du contrat domestique, in Burgat, F. (Ed.), Les Animaux d'élevage ont-ils droit au bien-être?, Paris, INRA Éditions, 9-24.

Larson, J., 2006. Disposal of Dead Production Animals, 19882006, Animal Welfare Information Center, United States Department of Agriculture, Agricultural Research Service, National Agricultural Library (http://www.nal.usda.gov/ awic/pubs/carcass.htm).

Mouret, S., 2005. Travailler en élevage industriel de porcs : «on s'y fait de toute façon c'est comme ça », Travailler, 14, 21-46.

Pigeon, S., Fortier, C., 2004a. Un projet pilote sur 16 sites. Composter les animaux morts, Porc Québec, octobre.

Pigeon, S., Fortier, C., 2004b. Trois incinérateurs d'animaux morts au banc d'essai, Porc Québec, juin.

Porcher, J., 2002. Éleveurs et animaux : réinventer le lien, Paris, PUF.

Porcher, J, 2003a. Bien-être et souffrance en élevage : conditions de vie au travail des personnes et des animaux, Sociologie du travail, 45, 27-43.

Porcher, J., 2003b. La Mort n'est pas notre métier, La Tour d'Aigues, L'Aube.

Rémy, C., 2003. Une mise à mort industrielle « humaine »? L'abattoir ou l'impossible objectivation des animaux, Politix, 16, 64, 53-73.

Rietveld, G., 2003a. Euthanasie à la ferme des moutons et des chèvres, Ontario, ministère de l'Agriculture, de l'Alimentation et des Affaires rurales (http://www.omafra.gov.on.ca/ french/livestock/animalcare/facts/ info_euthanasia_shgt.htm).

Rietveld, G., 2003b. Euthanasie à la ferme des bovins et des veaux, Ontario, ministère de l'Agriculture, de l'Alimentation et des Affaires rurales (http://www.omafra.gov.on.ca/ french/livestock/animalcare/facts/ info_euthanasia_cc.htm).

Salmona, M., 1985. Les Paysans français : le travail, les métiers, la transmission des savoirs, Paris, L'Harmattan.

Straus, E., 2000 (2 éd.). Du sens des sens : contribution à l'étude des fondements de la psychologie, Grenoble, J. Million.

Thomas, L.V., 1975. Anthropologie de la mort, Paris, Payot.

Vialles, N., 1987. Le Sang et la chair : les abattoirs des pays de l'Adour, Paris, MSH.

Viel, L., 2006. Le Biovator prêt à l'emploi, Porc Magazine, 400, 70.

Reçu le 24 avril 2006. Accepté le 3 mai 2007. 